\title{
Giant Raman Scattering from J-Aggregated Dyes inside Carbon Nanotubes for Multispectral Imaging
}

\author{
E. Gaufrès ${ }^{1}$, N.Y.W. Tang ${ }^{1}$, F. Lapointe ${ }^{1}$, J. Cabana ${ }^{1}$, M.A. Nadon ${ }^{1}$, N. Cottenye ${ }^{1}$, F. Raymond ${ }^{1}$, \\ T. Szkopek ${ }^{2}$ and R. Martel ${ }^{1 *}$
}

${ }^{1}$ Regroupement Québécois sur les Matériaux de Pointe and Département de Chimie, Université de Montréal, Montréal, Québec H3C 3J7, Canada

${ }^{2}$ Regroupement Québécois sur les Matériaux de Pointe and Department of Electrical and Computer Engineering, McGill University, Montréal, Québec H3A 2A7, Canada.

*Correspondence to: r.martel@umontreal.ca

Raman spectroscopy uses visible light to acquire vibrational fingerprints of molecules, thus making it a powerful tool for chemical analysis in a wide range of media. Its potential for optical imaging at high resolution is, however, severely limited by the fact that the Raman effect is weak. Here, we report the discovery of a giant Raman scattering effect from encapsulated and aggregated dye molecules inside single-walled carbon nanotubes (SWNTs). Measurements performed on rod-like dyes, such as $\alpha$-sexithiophene and $\beta$ carotene, assembled inside SWNTs as highly polarizable $\mathbf{J}$-aggregates indicate a resonant Raman cross-section (CS) of $\sim 10^{-21} \mathrm{~cm}^{2} / \mathrm{sr}$, which is well above the CS required for detecting individual aggregates at the highest optical resolution. Free from fluorescence background and photobleaching, this giant Raman effect allows the realization of a library of functionalized and biocompatible nanoprobe labels for Raman imaging with robust detection using multispectral analysis. 
Although Raman spectra display narrow bands containing powerful information for chemical analysis and detection, its use for high-resolution optical imaging remains marginal due to its inherent lack of sensitivity. The Raman cross-section, which relates the scattered intensity of a molecule to the incident power density of the light, is typically $10^{-28}-10^{-30} \mathrm{~cm}^{2}$ per molecule, which is too low for imaging and detecting individual objects at high resolution. This disadvantage is further compounded by the strong fluorescence background and photodegradation of chromophores that can accompany Raman spectroscopy.

Two main enhancement strategies, called Resonant Raman Spectroscopy (RRS) and Surface-Enhanced Raman Spectroscopy (SERS), have been pursued in the past in order to solve at least the sensitivity issue and to derive Raman nanoprobe labels (also called tags) for imaging applications ${ }^{1,2}$. However, each has shown significant limitations. For RRS, the optical resonances in dye molecules such as Rhodamine 6G, which are conveniently located in the visible spectrum, can boost the cross section to $\sim 10^{-24} \mathrm{~cm}^{2}$ per molecule, but making Raman dye labels is hardly practical due to stability issues, especially photobleaching, and to the presence of a strong fluorescence background ${ }^{2,3}$. Approaches based on SERS can increase further the CS by coupling with plasmonic resonances in metallic nanostructures, leading to an immense but local enhancement of the electromagnetic field ${ }^{1,2,4,5}$. The success of this approach is evident by the fact that the SERS cross-section of resonant dyes can reach up to $\sim 10^{-18} \mathrm{~cm}^{2}$ per molecule, a value that is close to the CS of fluorescence. The SERS effect has been successfully implemented to the design of Raman nanoprobes, but the specific requirements in size, shape (enabling hot spots by interparticle geometry) and composition (typically $\mathrm{Ag}, \mathrm{Au}$ ) for the SERS antennas add stringent design constraints and stability issues. Furthermore, the occurrence of hot spots in SERS gives inhomogeneous enhancements that greatly complicate quantitative analysis. 
Here, we report the discovery of giant resonant Raman effects from J-aggregated dyes encapsulated inside single-walled carbon nanotubes (SWNTs). The scattering intensities of the dyes combined with the suppression of background fluorescence and photobleaching provide robust spatial and multispectral detection of a single assembly in the limits of optical resolution. We elucidate the origin of this Raman effect and demonstrate its use to design a new library of stable, yet chemically tunable, Raman nanoprobes (also called tags) for experiments in Raman imaging and labeling. Multispectral Raman imaging demonstrations of the dyes-SWNT nanoprobes deposited on surfaces and attached onto yeast cells are also presented.

For our study, well-known organic dyes, such as $\alpha$-sexithiophene (6T) and $\beta$-carotene ( $\beta$ car), were encapsulated inside SWNTs having $\sim 1.3 \pm 0.2 \mathrm{~nm}_{\text {diameter }}{ }^{6}$. As schematized in Figure 1, a three-step procedure was followed to fabricate the dye@SWNTs in solution: 1) Purification (oxidation) and cutting of SWNTs; 2) encapsulation of the dyes inside SWNTs (giving the 6T@SWNTs); 3) covalent functionalization (labeled as6T@f-SWNTs). Only a detailed Raman study on 6T encapsulated in SWNTs (6T@SWNTs) is presented below, but the same conclusions can be drawn with $\beta$ car@SWNTs and other dyes tested so far. The detailed characterization of the dye@SWNT systems prepared by our method is consistent with results from other groups on 6T@SWNTs ${ }^{7-11}$ and $\beta$ car@SWNT ${ }^{12,13}$ in bulk form and clearly indicates that the dyes have been encapsulated. See supplemental information file for details.

\section{Figure 1 | Schematic representation of 6T@f-SWNT.}


The presence of optical resonances was established by the acquisition of absorption spectra of bulk SWNTs,6T@SWNTs and6T@f-SWNTs dispersed in mineral oil (Fig. 2a). The absorption of the 6T dyes in the 6T@SWNT spectrum is clearly seen as an intense and structured band in the wavelength range between $350-700 \mathrm{~nm}$. Additional weak SWNT absorption bands are observed, overlapping in part with the $6 \mathrm{~T}$ band.

In order to avoid averaging effects and other optical phenomena such as energy transfer between adjacent6T@SWNTs, Raman measurements at $\lambda=532 \mathrm{~nm}$ wavelength excitation were taken on isolated SWNTs deposited on an $100 \mathrm{~nm}$ thick oxide grown on a silicon substrate $\left(\mathrm{SiO}_{2} / \mathrm{Si}\right)$. An isolated $\mathrm{SWNT}$, single in appearance, with $\sim 1.4 \mathrm{~nm}$ diameter was located using atomic force microscopy (AFM) and probed using micro-Raman spectrometry (Supplementary Fig. S2 and S3). Raman spectra were then acquired using the same polarization and position conditions (located using markers patterned by optical lithography) after each step of the preparation, as in Figure 1. Before encapsulation (top spectrum, Fig. 2b), a strong Raman peak at $\sim 1590 \mathrm{~cm}^{-1}$ was seen and ascribed to the tangential vibrational mode (TM) of the SWNT. After encapsulation (middle spectrum, Fig. 2b), another Raman feature appeared at $1450 \mathrm{~cm}^{-1}$. This feature was unambiguously identified as being the $\mathrm{C}=\mathrm{C}$ stretching mode vibrating along the main axis of the $6 \mathrm{~T}$ molecules ${ }^{14}$.

Additional polarization experiments at $532 \mathrm{~nm}$ were done on the same individual 6T@SWNT (see Fig. 3c), where the polarization angle $\Omega$ is defined using the polarization vector of the incident light and the orientation of the nanotube axis. The superposition of a $\cos ^{2} \Omega$ dependency over the polarization-dependent intensity of the $6 \mathrm{~T}$ mode at $1450 \mathrm{~cm}^{-1}$ and the SWNT mode at $1590 \mathrm{~cm}^{-1}$ demonstrates that the transition dipole moments of the molecules are aligned together. Thus a $6 \mathrm{~T}$ aggregate is clearly formed with polarizability tensors, $\alpha_{\mathrm{ij}}$, perfectly 
oriented along the nanotube axis ${ }^{7}$. This result is consistent with the structure of 6T@SWNT illustrated in Figure 1a, which was previously deduced from transmission electron microscope images and calculations ${ }^{8,9}$. That is, the $6 \mathrm{~T}$ molecules are stacked in pairs and aligned head-to-tail, thus forming J-type aggregates inside the nanotube.

The detection of a Raman signal from the encapsulated $6 \mathrm{~T}$ molecules in an isolated SWNT is unexpected because only a few hundreds of molecules (455 at maximum filling) are present within the $500 \mathrm{~nm}$ focus spot of our Raman instrument. Indeed, surfactant molecules and even large polymers attached to the nanotube sidewalls are generally not detected in Raman microspectrometry. For further comparison, $6 \mathrm{~T}$ aggregates having $40 \mathrm{~nm}$ in diameter and located on $\mathrm{SiO}_{2} / \mathrm{Si}$ but far away from the SWNTs were probed using Raman and AFM. As expected, sub-micrometer aggregates and even a $50 \mathrm{~nm}$ thick film of $6 \mathrm{~T}$ gave much weaker signals and strong fluorescence backgrounds (Supplementary Figs S6 and S7 and Table S1). We therefore conclude that the Raman signal from the 6T@SWNT is strongly enhanced and arises from an encapsulated $6 \mathrm{~T} \mathrm{~J}$-aggregate, and not from other $6 \mathrm{~T}$ aggregates on the oxide surface.

Significantly, only minimal fluorescence at $\lambda=532 \mathrm{~nm}$ was detected. Both results are consistent with previous work on bulk6T@SWNTs in solution, although some fluorescence was detected but at shorter excitation wavelengths ${ }^{8,9}$. Other studies on different dyes inside SWNT reported efficient fluorescence quenching by the SWNTs ${ }^{12,13,15}$ and this effect seen in weakly bonded chromophore-SWNT complexes was explored recently in the context of light harvesting applications ${ }^{16,17}$.

We determined the Raman CS of encapsulated 6T aggregates using the SWNT signal as an internal reference. Using a laser excitation at $\lambda=532 \mathrm{~nm}$, a RRS condition for both $6 \mathrm{~T}$ and SWNTs, and the highest spatial resolution $(\sim 500 \mathrm{~nm})$ of our microscope, the Raman bands of the 
6T at $1450 \mathrm{~cm}^{-1}$ and of the SWNT at $\sim 1590 \mathrm{~cm}^{-1}$ exhibited nearly the same intensity.

Considering that the RRS CS at the TM mode of an individual SWNT is $\sim 10^{-21} \mathrm{~cm}^{2} / \mathrm{sr}^{18-20}$, we estimate that the cross section of the $6 \mathrm{~T}$ J-aggregate is roughly the same, i.e. $\sim 10^{-21} \mathrm{~cm}^{2} / \mathrm{sr}$. This comparison thus provides a fair estimate of the dye CS, assuming that the $6 \mathrm{~T}$ has filled the volume of the nanotube. A complete filling of the nanotube with the 6T J-aggregate is, however, unlikely given the liquid-phase procedure used in this study for the encapsulation. This Raman CS value is thus only a lower bound (the CS of incoherent Raman scattering scales linearly with the number of molecules) and clearly demonstrates a giant scattering effect in Raman from the 6T J-aggregate inside the SWNT. Using RRS data mentioned above on amorphous aggregates and on thin films of 6T molecules on $\mathrm{SiO}_{2} / \mathrm{Si}$, the normalized signal of the 6T@SWNT compared to other similarly close packed $6 \mathrm{~T}$ aggregates is roughly $10^{4}$ times more intense (Supplementary file Table S1).

\section{Figure 2 | Absorbance and Raman spectra of SWNTs, 6Ts, 6T@SWNTs and 6T@f- SWNTs.}

To understand the origin of the enhancement of the Raman CS, we investigated the influence of the SWNT resonances on the Raman scattering from the dyes and tested the hypothesis that strong optical resonances in the SWNT can produce an optical antenna effect similar to a SERS mechanism. That is, the scattering efficiency can be reduced or enhanced by the optical near field at the interior of the SWNT. The strength of this effect was estimated by modeling the SWNT as a hollow cylinder with an infinitesimal wall characterized by an optical frequency conductance $g^{21}$. By applying Mie theory (see Supplementary for details and Fig S9), 
it can be shown that $E_{\text {int }} / E_{0}$, the ratio of the optical electric field in the interior of the SWNT to the illuminating plane wave, is

$$
\frac{E_{\mathrm{int}}}{E_{0}}=\left(1-\frac{i g Z \cdot H_{0}^{(1)}(k a) \cdot J_{0}(k a)}{H_{0}^{(1)}(k a) \cdot J_{0}^{\prime}(k a)-H_{0}^{\prime(1)}(k a) \cdot J_{0}(k a)}\right)^{-1},
$$

where $k=2 \pi / \lambda, a$ is the SWNT radius, $Z$ the impedance of free space, and the usual cylindrical Bessel function notation is used. A tube radius of $a=0.6 \mathrm{~nm}$ and a peak optical conductance $g=$ $8 e^{2} / h$ (i.e. in resonance), the field at the interior of the SWNT at an incident wavelength $\lambda=633$ $\mathrm{nm}$ is $E_{\text {int }} / E_{0}=0.999$, corresponding to a negligible screening of the optical near field. This estimate shows that the small SWNT diameter, as compared to the optical wavelength, renders optical enhancement or screening ineffective in the visible spectrum.

\section{Figure 3 | Polarized micro-Raman spectroscopy on individual SWNT.}

Our experiments come in support to this calculation and rule out the antenna effect from the SWNT as being the cause of the giant Raman effect from the dye J-aggregate. In our samples, two different families of nanotubes, the semiconducting (s-SWNT) and metallic (mSWNT) species, are present. The optical resonances can be excited with laser lines at $\lambda=633 \mathrm{~nm}$ for the $\mathrm{m}-\mathrm{SWNTs}$ and at $\lambda=532 \mathrm{~nm}$ and $488 \mathrm{~nm}$ for the s-SWNTs. Using AFM, two individual SWNT specimens were selected and subsequently probed in situ using Raman microspectrometry before and after encapsulation. In Figure 3 , the RRS spectra taken at $\lambda=633$ nm, $532 \mathrm{~nm}$ and $488 \mathrm{~nm}$ for the two 6T@SWNTs confirmed that one nanotube is metallic (Fig. 3a) whereas the other is semiconducting (Fig. 3b). At $\lambda=532 \mathrm{~nm}$ and $488 \mathrm{~nm}$, the individual 
6T@m-SWNT spectrum showed an intense peak from the 6T molecules at $1460 \mathrm{~cm}^{-1}$ and almost no signal from the m-SWNT. Consistent with the absorption spectra in Figure $2 \mathrm{a}$, the Raman signal of the m-SWNT is off resonance while it is clearly in resonance for the 6T aggregates. At $\lambda=633 \mathrm{~nm}$, the opposite situation is observed: The signal is weak for the $6 \mathrm{~T}$ molecules and strong for the m-SWNT. The Raman scatterings of the m-SWNT and 6T are therefore uncorrelated and simply follow their respective resonance profiles. For the individual 6T@sSWNT (Fig. 3b), the Raman signatures from both constituents are seen at $\lambda=488 \mathrm{~nm}$ and 532 $\mathrm{nm}$, but not at $\lambda=633 \mathrm{~nm}$, indicating that the $6 \mathrm{~T}$ scattering intensity is only correlated to the resonance of the $6 \mathrm{~T}$ aggregates. Whether the SWNT is metallic or semiconducting, in resonance or not, has no observable influence on the scattering of the encapsulated 6T. This finding indicates that the reported giant CS is a robust phenomenon that depends only on the optical properties of the confined dye aggregates. As discussed below, this is a key feature enabling a whole new set of applications with functionalized dyes@SWNT nanoprobes for multispectral Raman labeling and imaging.

All of our experiments point toward an enhancement mechanism that is driven by RRS and J-type aggregation induced by confinement. The Raman signal of the dyes was also found to be stable against photobleaching, even at high laser fluence and free from fluorescence. By considering that 455 molecules of $6 \mathrm{~T}$ is the maximum number that can fit inside a $500 \mathrm{~nm}$ long SWNT (i.e. the diameter of our laser spot) and assuming incoherent scattering, a RRS CS of $\sim 5 \times 10^{-24} \mathrm{~cm}^{2} / \mathrm{sr}$ per $6 \mathrm{~T}$ molecule can be deduced. Literature data for the $6 \mathrm{~T}$ was not found, but this value corresponds to the RRS CS of dyes such as Rhodamine $6 \mathrm{G}^{3}$ and $\beta$-carotene ${ }^{22,23}$ in solution, and matches the CS of Rhodamine 6G physisorbed on a graphene layer where fluorescence is also quenched ${ }^{24}$. The cooperative effect of many molecules aligned as a J- 
aggregate inside SWNT boosts, however, the total cross section to $\sim 10^{-21} \mathrm{~cm}^{2} / \mathrm{sr}$ per dyes@SWNT assembly in the laser focal point, or more conveniently to a value of $\sim 2 \times 10^{-24}$ $\mathrm{cm}^{2} / \mathrm{sr}$ for each nanometer of filled SWNT. This remarkably large cross section has important implications for Raman imaging since a shorter segment of the dye aggregate, at least shorter than the optical resolution limit of our instrument $(<500 \mathrm{~nm})$, can be readily detected using conventional Raman microspectrometry.

\section{Figure 4 | Demonstration of dyes@SWNTs as Raman nanoprobes.}

Another main requirement in the elaboration of a competitive nanoprobe for imaging is its covalent functionalization. We probed the influence of chemical functionalization of 6T@SWNTs using a radical reaction that covalently attaches bromophenyl moieties to the nanotube sidewall. This is an important step toward achieving the dispersion, chemical functionality or selectivity and biocompatibility of the 6T@SWNT nanoprobes. In Figure 2a, the bulk6T@f-SWNT products in mineral oil exhibits an absorption spectrum that is similar to that of6T@SWNT, except for a significant weakening of the SWNT absorption bands. The Raman spectrum taken after the functionalization of the individual semiconducting 6T@SWNT of Figure $2 \mathrm{~b}$ is shown at the bottom. After functionalization, the TM-band of the SWNT at $\sim 1590$ $\mathrm{cm}^{-1}$ decreased in intensity, which is related to disorder induced into the carbon lattice by the covalent bonding of the bromophenyl grafts ${ }^{25}$. The fluorescence quenching ability of the fSWNT is preserved, as no fluorescence was observed in the spectra. More importantly, the Raman intensity of the $6 \mathrm{~T}$ modes remained constant, which indicates that the SWNT sidewall acts as a barrier that protects the dyes ${ }^{12}$ and their giant scattering CS. 
Experiments on a variety of rod-like dyes indicate that the giant CS observed in the 6T@f-SWNT system is common to a large family of chromophores. For instances, methylene violet B (MV), 3,3'-dimethylthiadicarbocyanine iodide (DTDCI) and oligothiophene derivatives such as 3,6-Bis-[2,2']bithiophenyl-5-yl-2,5-di-n-oc-tylpyrrolo[3,4-c]pyrrole-1,4-dione (DPP2) encapsulated inside SWNTs gave similar RRS responses but at a lower wavelength of $\lambda=633$ nm (Figs. 4d). These general properties of dye@SWNT nano-assemblies thus make them well adapted for constructing Raman nanoprobes for imaging and tagging experiments. For the purpose of demonstrating the applicability of the Raman effect, we tested various nanoprobes in different imaging configurations. First a sub-monolayer of 6T@SWNTs deposited on a $\mathrm{SiO}_{2} / \mathrm{Si}$ substrate was patterned and then imaged using SEM (Fig. 4a) and micro-Raman mapping (Fig. 4b). The Raman acquisition time at $300 \mu \mathrm{W} / \mu \mathrm{m}^{2}$ laser power density was only five seconds per pixel. The Raman 6T signal correlates with the varying density of nanoprobes observed using SEM (Fig. 4c), indicating that the signal is probably proportional to the density of nanoprobes. In another experiment shown in Figure 5a, a mixture of isolated $\beta$ car@SWNTs and 6T@SWNTs nanoprobes were deposited on $\mathrm{SiO}_{2} / \mathrm{Si}$ and subsequently analyzed by hyperspectral Raman imaging at $\lambda=532 \mathrm{~nm}$ excitation. Thanks to the sharp and specific vibrational signatures of each nanoprobes, the 6T@SWNTs (red) and the $\beta$ car@SWNTs (green) signatures were readily and unambiguously detected and selectively localized on the surface without interference from each other. The presence of a third dyes@SWNT in Figure 5b made with phenazine molecules (Ph@SWNTs in blue, see supplementary file Fig. S8b) was also detected, which further illustrates the powerful application of multispectral bands in identifying nanoprobes, even when they are overlapping at the same location. 
It is important to mention here that SWNTs are already known as giant Raman scattering objects exhibiting RRS cross-section between $10^{-22}-10^{-21} \mathrm{~cm}^{2} / \mathrm{sr}$ depending on the bands ${ }^{18-20}$. They also produce no fluorescence in the visible spectrum, which is a key feature enabling Raman applications. In effect, Dai and coworkers have shown that functionalized SWNTs can be used as Raman labels for protein detection ${ }^{26}$ and biological imaging of cancer cells ${ }^{27}$. As Raman nanoprobes, SWNTs are therefore interesting, but they lack, however, of versatility because they provide a small number of Raman-active bands, which limits differentiation between nanotube species. Also, SWNTs having different chiralities operate at different RRS energies, which implies that SWNT labels require fastidious sorting on the basis of their resonance energies. Furthermore, adding chemical functions onto the SWNT sidewalls by covalent reaction for dispersion in liquids and biocompatibility drastically reduces their Raman CS. In this context, the giant Raman CS reported here on J-aggregated dyes inside SWNTs is an important step forward that circumvents the hurdles of using the SWNT signal only for detection. Last, the multiplexing of the Raman signals as shown above is similar to that of Raman labels made with functionalized SWNTs having different contents of ${ }^{13} \mathrm{C} /{ }^{12} \mathrm{C}$ isotopes ${ }^{26,27}$. However, the number of combinations that can be derived with dye@f-SWNT is, by far, much greater owing to the large number from dyes and their narrow bandwidth in the Raman spectra.

Finally we show preliminary results on the biocompatibility of dyes@f-SWNT and their use as contrast agent for living cells. $\beta$ car@SWNTs was functionalized using poly(ethylene glycol) bis-(3-aminopropyl) (PEG) to allow their solubility in physiological saline solution and mixed with a culture of a yeast called Candida Albican (see Method and Supplementary file for details). The Figure $5 \mathrm{~b}$ (left) presents an optical image of aggregates of Candida Albican cells mounted on a glass slide after tagging with $\beta$ car@f-SWNTs and rinsing with saline solution. The 
Raman hyperspectral image taken at the same place (Fig. 5b right) shows that the tagged yeasts scatter strongly at the characteristic Raman frequency of the $\beta$ car@f-SWNT nanoprobes. The uniform contrast across the cells indicate also that the $\beta$ car@f-SWNT nanoprobes have bonded to the cell membranes and some may have penetrated into the yeasts. Importantly, the tagged yeasts lived more than 3 weeks after their incubation with $\beta$ car@f-SWNT, which is preliminary indication of the low toxicity of the dyes@f-SWNTs.

In summary, the dye@f-SWNT nano-assembly gives access to a giant resonant Raman scattering effect that is powerful for multispectral Raman imaging experiments. Our study shows that the SWNT is in essence a capsule that not only quenches fluorescence, but acts also as a template for J-aggregation as well as a protection of dye molecules from the environment. By adding chemical functions to the SWNT sidewalls and by expanding the variety of encapsulated dyes exhibiting such giant Raman scattering, outstanding opportunities can be created with the dyes@f-SWNT nano-assemblies for experiments requiring a large library of robust and nanometer size Raman tags.

\section{Methods}

The single-wall carbon nanotubes (SWNTs) used in this work were produced by laser and provided by Benoit Simard from the National Research Council of Canada. The SWNT were first purified in concentrated nitric acid, washed with water and dispersed in dimethylformamide (DMF) for encapsulation. The encapsulation of the dyes was performed under reflux by mixing $10 \mathrm{mg}$ of the dye in $10 \mathrm{~mL}$ of an appropriate organic solvent with $10 \mathrm{mg}$ of purified SWNTs. For the study of individual SWNT, the SWNT were dispersed in DMF and deposited onto $\mathrm{SiO}_{2} / \mathrm{Si}$ substrate coated with an aminopropyltriethoxysilane (APTES) layer. This substrate was annealed 
under vacuum at $500{ }^{\circ} \mathrm{C}$ for $1 \mathrm{hr}$, and then immersed in a solution of dyes (at $1 \mathrm{mg} / \mathrm{mL}$ ) of organic solvent. The solution was then heated under reflux for 24 hours followed by rinsing in DMF. Atomic force microscopy and polarized micro-Raman spectroscopy were used to characterize these encapsulated SWNTs.

The sample for the multispectral imaging was prepared by depositing the Ph@SWNT, 6T@SWNT and $\beta$ car@SWNT on a $\mathrm{SiO}_{2} / \mathrm{Si}$ substrate coated with an APTES layer. The preparation of the Candida Albican tagged with $\beta$ car@SWNT required the functionalization of SWNTs with poly(ethylene glycol) bis-(3-aminopropyl $)^{28}$ in order to obtain a water soluble nanoprobes. The solvated nanoprobes were then incubated with Candida Albican yeast broth ${ }^{29}$ during $30 \mathrm{~min}$, rinsed and centrifuged before dispersing again in physiological saline. The yeasts were finally mounted on a glass slide for imaging. The Raman images were done using a hyperspectral Raman imager RIMA ${ }^{\mathrm{TM}}$ from Photon etc ltd. The experimental procedures and materials are described in details in the supplementary information file.

\section{REFERENCES}

[1] Le Ru, E. C. \& Etchegoin, P. G. Principle of surface-enhanced Raman spectroscopy (Elsevier, Oxford, UK 2009).

[2] Aroca, R. Surface-enhanced vibrational spectroscopy (J. Wiley \& Sons, Ltd West-Sussex, UK, 2006).

[3] Shim, S. Stuart, C. M. Mathies, R. A. Resonance Raman cross-sections and vibronic analysis of Rhodamine 6G from broadband stimulated Raman spectroscopy. ChemPhysChem 9, 697-699 (2008). 
[4] Stiles, P. L. Dieringer, J. A. Shah, N. C. Van Duyne, R. P. Surface-enhanced Raman spectroscopy. Annu. Rev. Anal. Chem. 1, 601-626 (2008).

[5] Moskovits, M. Surface-enhanced spectroscopy. Rev. Modern Phys. 57, 783-826 (1985).

[6] Kingston, C. T. Jakubek, Z. J. Dénommé, S. \& Simard, B. Efficient laser synthesis of singlewalled carbon nanotubes through laser heating of the condensing vaporization plume. Carbon 42, 1657-1664 (2004).

[7]Hasobe, T. Fukuzumi S. \& Kamat, P. V. Ordered Assembly of Protonated Porphyrin Driven by Single-Wall Carbon Nanotubes: J- and H-Aggregates to Nanorods. J. Am. Chem. Soc. 127, 11884-11885 (2005).

[8] Loi, M. A. et al. Encapsulation of conjugated oligomers in single-walled carbon nanotubes: Towards nanohybrids for photonic devices. Adv. Mater. 22, 1635-1640 (2010).

[9] Gao, J. et al. Electronic interactions between "Pea" and "Pod": The case of oligothiophenes encapsulated in carbon nanotubes. Small, 7, 1807-1815 (2011).

[10] Kalbac, M. Kavan, L. Gorantla, S. Gemming, T. \& Dunsch, L. Sexithiophene encapsulated in a single-walled carbon nanotube: An in situ Raman spectroelectrochemical Study of a Peapod Structure. Chem. Eur. J. 16, 11753-11759 (2010).

[11] Alvarez, L. et al. Charge transfer evidence between carbon nanotubes and encapsulated conjugated oligomers. J. Phys. Chem. C 115, 11898-11905 (2011).

[12] Yanagi, K. Miyata, Y. \& Kataura, H. Highly stabilized $\beta$-carotene in carbon nanotubes. $A d v$. Mat. 18, 437-441 (2006). 
[13] Yanagi, K. et al. Light-harvesting function of $\beta$-carotene inside carbon nanotubes. Phys. Rev. B 74, 155420:1-5 (2006).

[14] Esposti, A. D. Fanti, M. Muccini, M. Taliani, C. \& Ruani, G. The polarized infrared and Raman spectra of $\alpha$-T6 single crystal: An experimental and theoretical study. J. Chem. Phys. 112, 5957-5969 (2000).

[15] Takenobu, T. et al. Stable and controlled amphoteric doping by encapsulation of organic molecules inside carbon nanotubes. Nature Mat. 2, 683-688 (2003).

[16] Garrot, D. et al. Time-resolved investigation of excitation energy transfer in carbon nanotube-porphyrin compounds. J. Phys. Chem. C 115, 23283-23292 (2011).

[17] Huang, C. et al. Spectroscopic properties of nanotube-chromophore hybrids. ACS Nano 5, 7767-7774 (2012).

[18] Bohn, J. E. et al. Estimating the Raman Cross Sections of Single Carbon Nanotubes. ACS Nano 4, 3466-3470 (2010); note that a CS value between $1-4 \times 10^{-22} \mathrm{~cm}^{2} / \mathrm{sr}$ is reported for the radial breathing mode (RBM), but the TM band (or G-band) is about 10 times more intense.

[19] Jorio, A. et al. Structural (n, m) determination of isolated single-wall carbon nanotubes by resonant Raman scattering. Phys. Rev. Lett. 86, 1118-1121 (2001).

[20] Duesberg, G. S. Loa, I. Burghard, M. Syassen K. \& Roth, S. Polarized Raman Spectroscopy on Isolated Single-Wall Carbon Nanotubes. Phys. Rev. Lett. 85, 5436-5439 (2000).

[21] Joh, D. Y. et al. Single-walled carbon nanotubes as excitonic optical wires. Nat. Nanotechnol 6, 51-56 (2011). 
[22] OuYang, S. L. et al. Effect of the structural order of all-trans- $\beta$-carotene on the Raman scattering cross section at low concentrations. J. Raman Spectrosc. 41, 1650-1654 (2010).

[23] Tian, Y.J. et al. Study of resonance Raman cross section of aqueous $\beta$-carotene at low concentrations. Appl. Phys. B 87, 727-730 (2007).

[24] Thrall, E. S. Crowther, A. C. Yu, Z. \& Brus L. R6G on graphene: High Raman detection sensitivity, yet decreased Raman cross-section. Nano Lett. 12, 1571-1577 (2012).

[25] Cabana, J. Lavoie S. \& Martel R. Thermal chemistry of methylene- and phenylfunctionalized carbon nanotubes. J. Am. Chem. Soc. 132, 1389-1394 (2010).

[26] Chen Z. et al. Protein microarrays with carbon nanotubes as multicolor Raman labels. Nat. Biotech. 26 1285-1292 (2008).

[27] Liu Z. et al. Multiplexed Five-Color Molecular Imaging of Cancer Cells and Tumor Tissues with Carbon Nanotube Raman Tags in the Near- Infrared. Nano Research 3, 222-233 (2010).

[28] Qi, H. et al. Functionalization of Single-Walled Carbon Nanotubes with Protein by Click Chemistry as Sensing Platform for Sensitized Electrochemical Immunoassay. Electrochimica Acta 63, 76-82 (2012)

[29] Vadeboncoeur, C., Mayrand, D. \& Trahan, L. A Comparative Study of Enzymes Involved in Glucose Phosphorylation in Oral Streptococci. J Dent Res 61, 60-65 (1982).

\section{ACKNOWLEDGMENTS}

The authors thank M. Côté, P. McBreen and C. Silva for useful discussions; B. Simard and his group for providing the SWNTs for this study; M. A. Loi and E. Menna for samples of 
6T@SWNTs and insightful suggestions; J. Barbeau for providing cultures of Candida Albican.

This work was possible because of financial supports from the Natural Sciences and Engineering Research Council of Canada, Nanoquébec and the Canada Research Chair programs.

\section{AUTHORS CONTRIBUTIONS}

Data were taken by and analyzed by E.G., N.T., F.L., J.C., M.A.N. and R.M. The experiments were conceived by E.G., N.T., J.C., F.L., M.A. N. and R.M. Calculations were carried out by T.S. Samples were prepared by N.T., E.G., N.C, F.L., F.R., J.C. and M.A.N. All authors contributed to the writing of the paper.

\section{FIGURE LEGENDS}

Figure 1 | Schematic representation of 6T@f-SWNT. Description of the encapsulation and chemical functionalization steps to prepare the $\alpha$-sexithiophene encapsulated inside a covalently functionalized single-walled carbon nanotube (6T@f-SWNT).

Figure 2 | Absorbance and Raman spectra of SWNTs, 6T, 6T@SWNTs and 6T@f-SWNTs. a, Absorbance spectra of bulk samples of SWNTs,6T,6T@SWNTs and6T@f-SWNTs dispersed in mineral oil at room temperature. The dotted lines correspond to the Raman laser lines: $\lambda=488 \mathrm{~nm}(2.54 \mathrm{eV}) ; \lambda=532 \mathrm{~nm}(2.33 \mathrm{eV}) ; \lambda=633 \mathrm{~nm}(1.96 \mathrm{eV})$. b. Raman spectra at $\lambda=$ $532 \mathrm{~nm}$ of an individual single-walled carbon nanotube (SWNT), of $\alpha$-sexithiophenes (6T) inside a SWNT (6T@SWNT) and after the covalent functionalization step (6T@f-SWNT). For clarity, the Raman spectra are shifted (by steps of 1). Peaks with * are instrumental artifacts.

Figure 3 | Polarized micro-Raman spectroscopy on individual SWNT. a-b, Raman spectra at three different laser lines (red: $1.96 \mathrm{eV}$ or $\lambda=633 \mathrm{~nm}$; green: $2.33 \mathrm{eV}$ or $\lambda=532 \mathrm{~nm}$; blue: 2.54 
$\mathrm{eV}$ or $\lambda=488 \mathrm{~nm}$ ) of an individual metallic 6T@m-SWNT (a) and of an individual semiconducting6T@s-SWNT (b). For clarity, the spectra have been shifted (baselines at 0,1.5 and 2.0). c, Variation in intensity of two Raman peaks (at $1590 \mathrm{~cm}^{-1}$ and $1440 \mathrm{~cm}^{-1}$ for the semiconducting SWNT and 6T modes, respectively) of an individual 6T@s-SWNT versus laser polarization angle $(\Omega)$ at $\lambda=532 \mathrm{~nm}$. The inset is an atomic force microscopy image of the SWNT and a description of the polarization angle.

Figure 4 | Demonstration of dyes@SWNTs as Raman nanoprobes. a, Scanning Electron Microscope image and $\mathbf{b}$, Raman intensity mapping at $\lambda=532 \mathrm{~nm}$ of the $6 \mathrm{~T}$ signal at $1450 \mathrm{~cm}^{-1}$ of patterned6T@SWNTs on a $\mathrm{Si} / \mathrm{SiO}_{2}$ substrate. c, A superposition of images (a) and (b).d, Examples of Raman spectra at $\lambda=532 \mathrm{~nm}$ and $\lambda=633 \mathrm{~nm}$ from different bulk samples of dyes@SWNTs prepared using a liquid phase procedure. From top to bottom, the encapsulated dyes are: 6T, $\beta$ car, DASPMI, DPP2, DTDCI and methylene violet B (see text). The spectra have been shifted for clarity.

Figure 5 | Multiplexing in Raman and bio-compatibility of dyes@SWNTs nanoprobes. a, Raman hyperspectral image at $\lambda=532 \mathrm{~nm}$ of isolated bundles of 6T@SWNTs (red) and $\beta$ car@SWNT (green) co-deposited at low coverage onto a $\mathrm{Si} / \mathrm{SiO}_{2}$ substrate. b, same as in (a), but using a mixture of 6T@SWNTs, $\beta$ car@SWNT and Ph@SWNT (blue) nanoprobes onto Si/SiO 2 . c, Optical image of Candida Albican tagged with $\beta$ car@f-SWNT functionalized with PEG. d, Raman image taken at $532 \mathrm{~nm}$ of the $\beta \mathrm{car} @ \mathrm{f}-\mathrm{SWNT}$ mode centered at $1520 \mathrm{~cm}^{-1}$. 
Figure 1

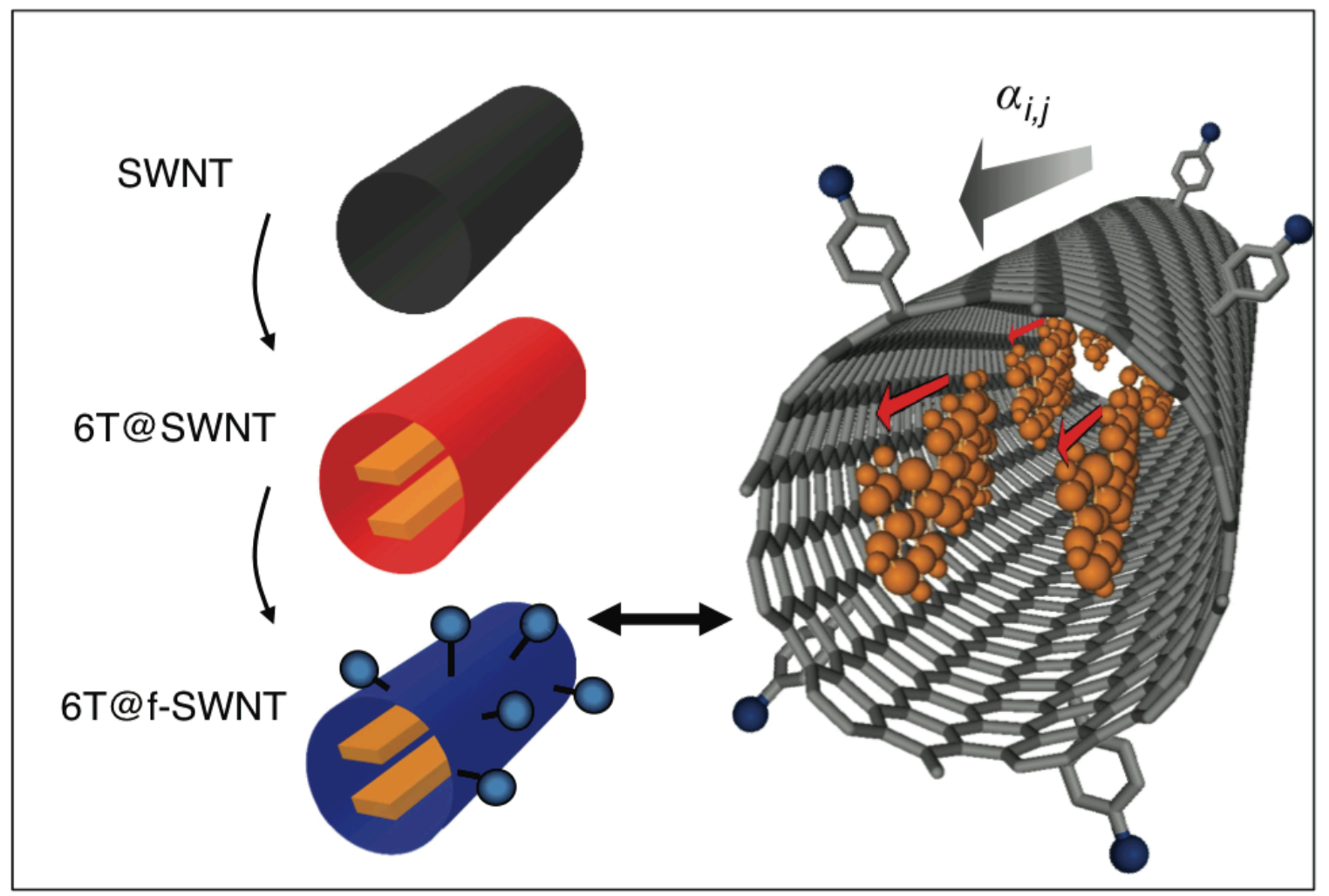


Figure 2
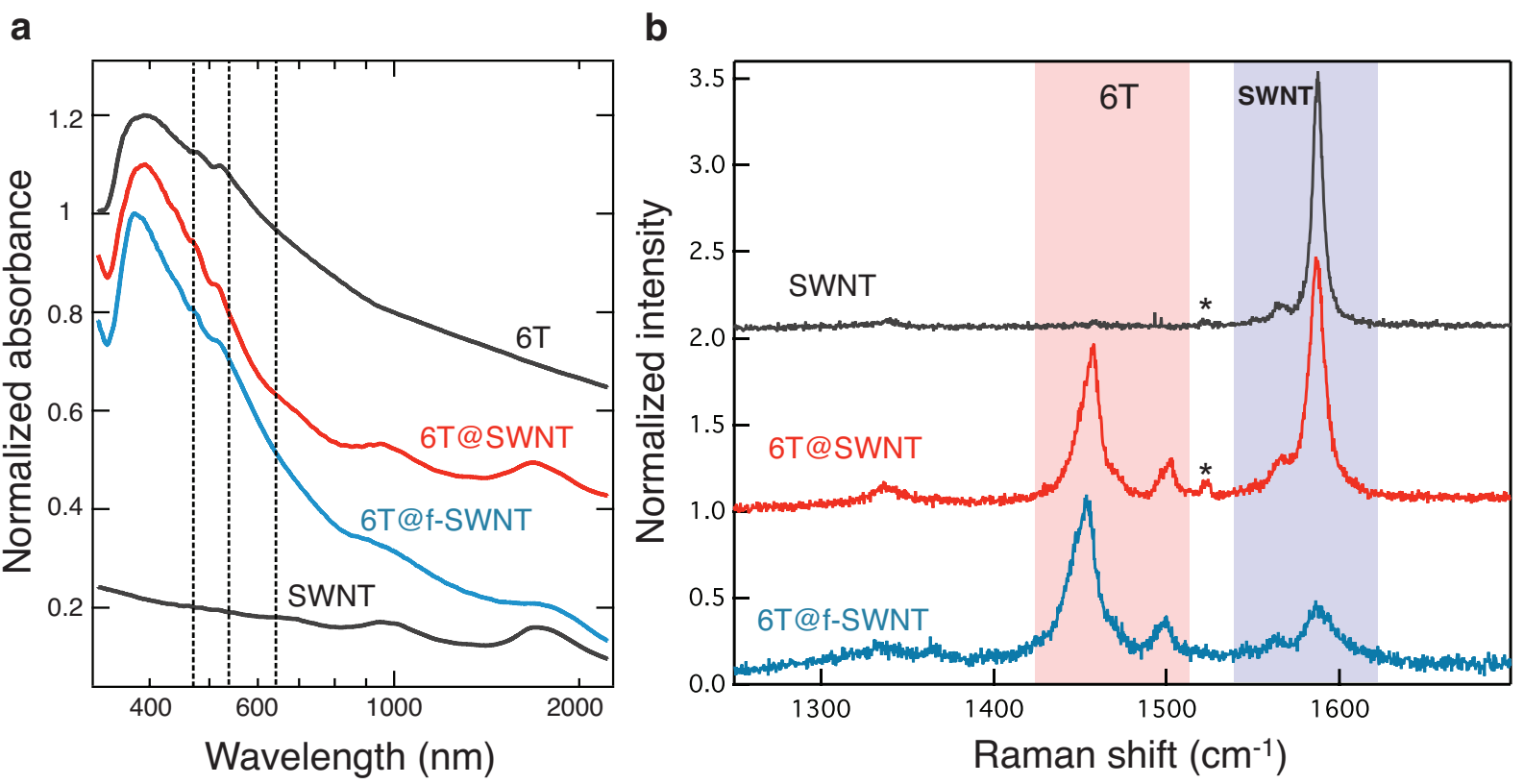
Figure 3
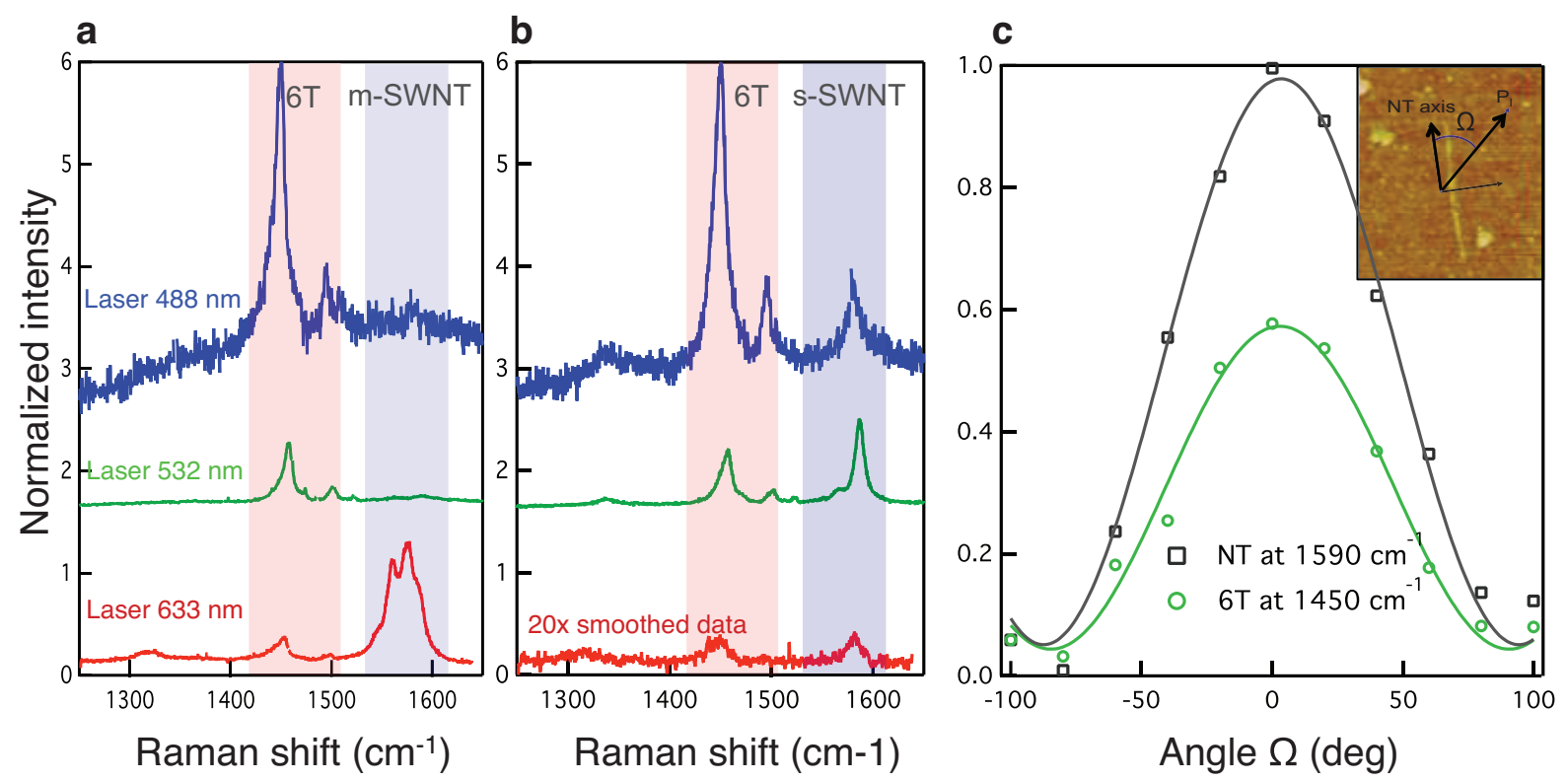
Figure 4
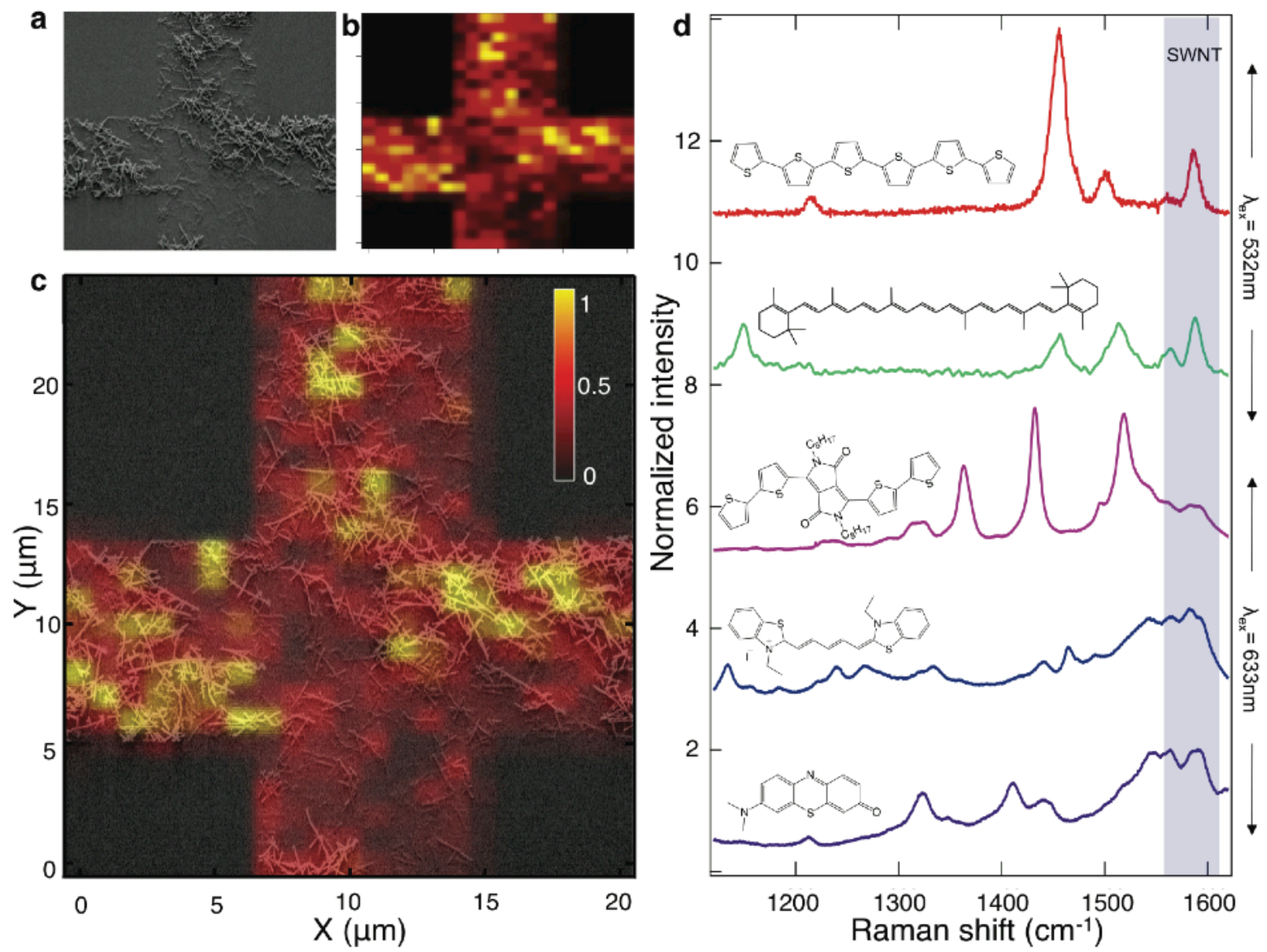
Figure 5

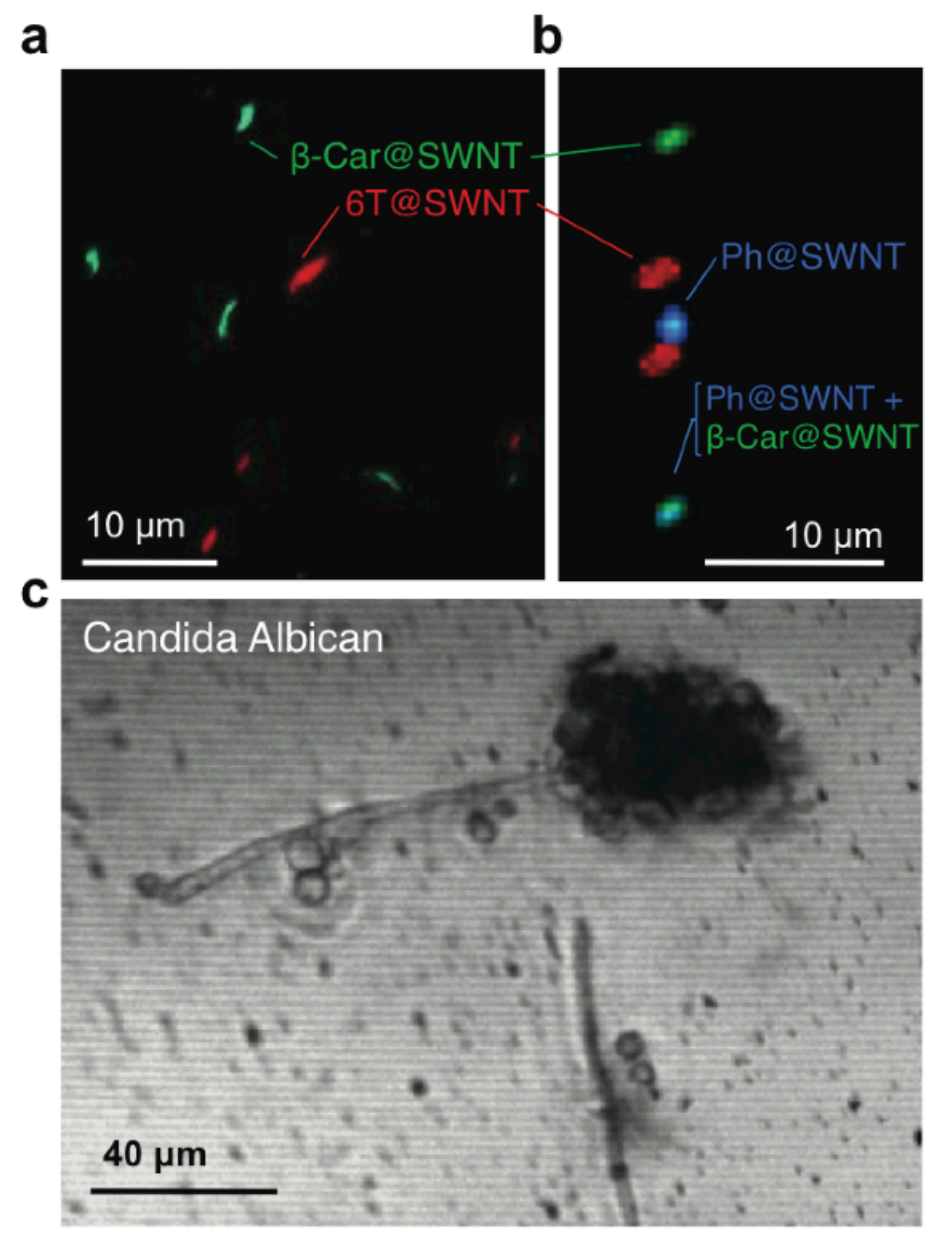

d $\beta$-Car@SWNT band at $1520 \mathrm{~cm}^{-1}$ 